\title{
DESARROLLANDO COMPETENCIAS DE INVESTIGACIÓN A TRAVÉS DE METODOLOGÍAS ACTIVAS EN UN ENTORNO B-LEARNING
}

\author{
José Sánchez-Santamaría \\ Sonia Morales Calvo \\ Universidad de Castilla La Mancha
}

\begin{abstract}
RESUMEN: El artículo muestra los resultados de un estudio sobre el uso de metodologías activas para el desarrollo de competencias en un entorno B-learning, en concreto, competencias profesionales de investigación en Educación Social. Para ello, en primer lugar, se procede a establecer una reflexión fundamentada sobre la enseñanza basada en competencias, en conexión con las metodologías activas y el B-learning; en segundo lugar, se realiza una delimitación y caracterización del perfil competencial del educador social; en tercer lugar, se aborda el contexto docente en el que se desarrollo la experiencia; en cuarto lugar, la metodología del estudio; en quinto lugar, se muestran los resultados obtenidos, en torno al grado de utilidad, desarrollo competencial percibido y satisfacción con el desarrollo de la asignatura. Y, por último, se recogen las conclusiones que, a modo de prospectiva, nos ayuden a orientar procesos futuros de reflexión y acción docente. Los resultados ponen de relieve el importante papel de las metodologías activas en B-learning para el desarrollo de competencias vinculadas a la investigación socioeducativa, al igual que permite un proceso de enseñanza-aprendizaje más continuo, progresivo y personalizado.
\end{abstract}

PALABRAS CLAVE: Docencia universitaria, enfoque basado en competencias, aprendizaje mixto, metodologías activas.

\section{PROMOTING THE DEVELOPMENT OF RESEARCH COMPETENCY THROUGH ACTIVE METHODOLOGIES IN B-LEARNING}

\footnotetext{
ABSTRACT: This article analyses the main findings of a study on the use of active methodologies for competency development in a B-learning, in particular, research professional competency in Social Education. To do this, a reflection on Competency-Based Education is proceed, in connection with active
} 
methodologies and B-learning; secondly, a delineation and characterization of the Social Educator's competence profile is carried out; Thirdly, the educational context in which development experience is addressed; fourth, the methodology of study is showed; fifth, the results on the degree of usefulness: perceived competence development and satisfaction with the development of the subject are analyzed. And finally, it describes the conclusions that, as a prospective, we help guide the further processes of reflection and action in teaching. The results highlight the important role of active methodologies in B-learning in the work of general, lateral and cognitive competencies, like allowing a teachinglearning process more continuous, progressive and personalized.

KEYWORDS: University teaching, Competency-Based Approach, blended learning, active methodologies.

Recibido: 17/08/2012

Aceptado: 25/01/2013

\section{INTRODUCCIÓN}

En un contexto de generación de nuevas demandas y necesidades para avanzar hacia la sociedad del conocimiento, la activación de procesos de innovación docente, favorecedores de metodologías activas (MEC, 2006), y el uso de Entornos Virtuales de Enseñanza y Aprendizaje (EVEA), son esenciales para ofrecer situaciones idóneas para el desarrollo competencial de los alumnos. En este sentido, las Tecnologías de la Información y la Comunicación (TIC) se han convertido en un elemento clave en los procesos de formación, gestión e investigación en muchas universidades del mundo, y en los últimos años, el sistema universitario español está experimentando un desarrollo sin precedentes (Uceda Antolín y Barro, 2009). Este aspecto se ha visto impulsado con la Estrategia 2015. Por lo que, la relevancia de los EVEA (Silva, 2011) está en que pueden actuar como "artefactos mediadores entre el docente y el alumnado o entre iguales que proporcionan un contexto educativo singular y virtual facilitador de procesos interactivos de co-construcción de conocimiento" (Salmerón, Rodríguez y Gutiérrez, 2010, p. 164). Un buen ejemplo de ello es la proliferación de experiencias de docencia universitaria basada en el modalidad 'blended learning' (B-learning) mediante el uso de 'Learning Management System' (LMS).

Por ello, la finalidad de este artículo se centra en describir y discutir los principales resultados de una experiencia de innovación docente, Ilevada a cabo en la asignatura de métodos de investigación educativa (MIE) del grado de Educación Social del Campus de Cuenca de la Universidad de Castilla-La Mancha (UCLM), durante el curso académico 2011/12; centrando la atención en la introducción de metodologías activas, para el desarrollo de competencias específicas de investigación, en un entorno B-learning. Este trabajo se enmarca en una línea de investigación más amplia sobre procesos de innovación educativa basados en la evidencia, que se concreta en: estudiar el desarrollo y evaluación de competencias profesionales vinculadas al 
desarrollo de la investigación socioeducativa; evaluar el impacto didáctico de las TIC; y, diseñar materiales didácticos orientados al aprendizaje situado basado en tareas auténticas. Además, se origina por la necesidad dar respuesta a los cambios que venimos experimentando por la reciente implantación del Espacio Europeo de Educación Superior (EEES), debido a la incorporación progresiva de nuevas estructuras organizativas y a las implicaciones curriculares y metodológicas de una enseñanza organizada en competencias (Morales y Sánchez-Santamaría, 2012).

Para dar contenido a la finalidad del artículo, en primer lugar, se procede a establecer una reflexión fundamentada sobre el papel del B-learning junto con la aplicación de metodologías activas; en segundo lugar, se realiza una delimitación y caracterización del perfil competencial del educador social; en tercer lugar, se aborda el contexto docente en el que se desarrollo la experiencia; en cuarto lugar, se establecen el método muestral y de estudio, así como el procedimiento de revisión por jueces, avalado por una experiencia piloto, para dar validez al cuestionario elaborado ad hoc; en quinto lugar, se muestran los resultados obtenidos, en torno a los aspectos metodológicos trabajados en la asignatura y a sus implicaciones para una toma de decisiones que permita introducir elementos de mejora basados en evidencias empíricas. Y, por último, se recogen las conclusiones que, a modo de prospectiva, nos ayuden a orientar procesos futuros de reflexión y acción docente.

\section{Enseñanza orientada al desarrollo de competencias profesionales EN LA UNIVERSIDAD: EL PAPEL DEL B-LEARNING}

Con el EEES se aprecia un cambio intrínseco del paradigma educativo, motivado por el desarrollo de las habilidades metodológicas, sociales, participativas y de los conocimientos de carácter práctico favorecedores de la aplicación de conocimientos teóricos (Ginés Mora, 2004). A ello, se unen las implicaciones organizativas, curriculares y metodológicas que este proceso está generando, y en los que se trata de que el estudiante ponga en acción un universo de saberes de tipo instrumental, interpersonal y sistemático (Villa y Poblete, 2007).

En este contexto, las TIC se han incorporado con fuerza en el proceso de enseñanza-aprendizaje en la Universidad. A parte de haber sido una política de integración impulsada por la Administración educativa para dar respuesta a las demandas formativas del EEES, siguiendo a Järvelä (2006, p. 40) se puede apreciar con claridad por qué las TIC son esenciales para el aprendizaje, en términos potenciales, a saber: a) aumento del grado de autenticidad del aprendizaje y el interés del alumnado; b) construcción de comunidades virtuales entre diferentes instituciones educativas, equipos colaborativos y profesorado; c) ayuda para compartir perspectivas entre estudiantes con distintos bagajes, promoviendo la ayuda entre iguales y las prácticas de referencia en diferentes campos; d) facilitación de la indagación mediada por la tecnología y los modelos de resolución de problemas para incrementar las habilidades de aprender a aprender; $y$, e) proporcionar formas innovadoras de integrar el apoyo sobre la marcha y las interacciones en diferentes contextos de aprendizaje. 
Entre los retos que plantea este nuevo escenario destacamos el de avanzar hacia la incorporación y combinación progresiva de metodologías activas, donde tecnologías favorecedoras de EVEA nos ofrecen cada vez más, mayores posibilidades pedagógicas (Bautista, Borges y Forés, 2006). Estos espacios pueden ser utilizados en procesos de e-learning, en los que el proceso de enseñanza aprendizaje es totalmente a distancia, o en procesos de B-learning, en los que la enseñanza presencial se complementa con sesiones o actividades dispuestas a través de un aula virtual sobre el LMS (Cole y Helen, 2007; Rodríguez Damián et al., 2009; Silva y Ramos, 2011).

La rápida incorporación del B-learning en la docencia universitaria (Alonso, López, Manrique y Viñes, 2005), la cual se desarrolla a través de campus virtuales de formación basados en Moodle, se debe a las posibilidades que oferta para la mejora de las condiciones de los procesos de enseñanza-aprendizaje en la Universidad, a saber (González-Videgaray, 2007): a) comunicación e interactividad: rompe la lógica exclusiva del "face to face"; b) accesibilidad: permite el acceso desde lugares muy lejanos y en cualquier momento; c) lo presencial asume una nueva forma de concebirse, lo virtual como espacio de formación es puede crear condiciones parecidas al aprendizaje situado; d) distribución: facilita el tránsito rápido y seguro de recursos educativos; e) en algunos casos, el entorno virtual puede ser algo vivo y dinámico generado por el alumno; f) responde a los ritmos de aprendizaje de los alumnos; g) entornos sólo accesibles a los alumnos y el profesor; y, h) favorece el desarrollo de e-competencias. Sin embargo, nos advierte que corremos el peligro de hacer un mal uso de las posibilidades que esta metodología docente nos ofrece, porque el aprendizaje on-line requiere de unas condiciones y recursos adecuados vinculados al diseño, contenido, herramientas de trabajo, apoyos del profesorado, percepciones de los alumnos, experiencias previas, etc.

\section{El perfil profesional del educador social}

\section{1. ¿Qué es un Educador Social?}

Se trata de una profesión de carácter pedagógico, siendo su ámbito de competencia, la generación de contextos educativos y acciones mediadoras y formativas para la incorporación del sujeto a la diversidad de las redes sociales y su promoción cultural y social (Riera Romaní, 1996; ASEDES, 2004). En este sentido, y desde una perspectiva más crítica, se entiende por educador social como aquel "profesional que interviene y es protagonista de la acción social conducente a modificar determinadas situaciones personales y sociales a través de estrategias educativas... se concibe como un agente de cambio social, dinamizador de los grupos y colectivos sociales a través de la acción educativa" (Petrus, 1994: 58). En torno a esta definición se alinea la de Mata Salvador (1998: 48): "el educador social es un agente de cambio social y un dinamizador de grupos sociales a través de la acción educativa".

Los ámbitos del educador social quedan recogidos a continuación, donde se realiza una comparación entre lo que se recoge en la literatura especializada sobre el tema y la opinión de los expertos consultados por la ANECA en la elaboración del Libro Blanco (tabla 1). 
Tabla 1. Ámbitos profesionales del Educador Social

\section{Literatura Especializada}

(Petrus, 1997, 2000; Luque, 1998; Fullana, 1999; Petrus, 2000; López Zaguirre, 2002; Maiztegui y Santibáñez, 2002; Serna Gómez, 2003; Ruiz, 2003)

\author{
ANECA \\ (Villa, 2004)
}

Ámbito de inserción social, orientando y mediando a los individuos o colectivos en situación de riesgo en su desarrollo personal, social y educativo
Marginación, drogodependencia y exclusión social

Intervención socioeducativa en menores Intervención educativa para el desarrollo comunitario y familiar

Ámbito de educación en centros escolares como PTFCSC, atendiendo a la alfabetización de alumnos extranjeros; mediando en la relación entre padres-profesores, profesores-alumnos y alumnos-alumnos, desarroIlando; y, diseñando, implementando y evaluando el programa de convivencia escolar y orientando el proceso de diversificación curricular de los alumnos

Atención socioeducativa a la diversidad

Ámbito de educación de personas adultas, diseñando programas de formación ocupacional y talleres de inserción social y siguiendo y atendiendo a las necesidades que se generen en el proceso de desarrollo Formación e inserción de personas adultas educativo de los individuos individualmente considerados

Ámbito socio-cultural, generando espacios y posibilitando herramientas a los diferentes colectivos de la población urbana y rural para que éstos posibiliten una mayor y meAnimación y gestión sociocultural jor participación ciudadana y cultural

Ámbito de educación ambiental, diseñando, implementando y evaluando programas de educación ambiental

Asimismo, también se recoge una propuesta de los contextos y funciones del Educador Social, a partir del conocimiento obtenido por la investigación (tabla 2). 
Tabla 2. Contextos de intervención profesional y funciones del Educador Social

\begin{tabular}{cc}
\hline Contextos & Funciones \\
\hline Socioeducativo & Intervención \\
Laboral & Orientación \\
Comunitario & Compensación \\
& Prevención \\
& Reeducación \\
\hline
\end{tabular}

Fuente: Villar, 2004, p.135.

En conclusión, cabe destacar que, en concordancia con lo expresado por Petrus (1997), el educador social es un agente de cambio social que toma como referente de su acción lo educativo, y se encuentra inmerso en un contexto cultural y socioeducativo en constante cambio que le requiere actualizarse, perfeccionarse y reflexionar sobre sí mismo y su práctica, bajo un objetivo común a otros profesionales, la mejora de la calidad de vida y del bienestar social de las personas. Así, la educación social como una profesión en construcción, relativamente joven, su profesionalización está en relación permanente a una serie de actores sociales con los cuales interactúa: grupo ocupacional, políticas sociales, universidad y mercado de trabajo (Sáez Carreras, 1994).

\section{2. ¿Qué papel juega la investigación en la intervención socioeducativa y desarrollo profesional del Educador Social?}

La práctica profesional del educador social se caracteriza por ser provisional, cambiante, dinámica y con una clara tendencia a hacerse innecesaria ya que se dirige a la superación de deficiencias, problemas y dificultades propias del desarrollo social y para la promoción y prevención social. Asimismo, viene determinada por el sujeto al que se dirige la acción socioeducativa, y por el modelo que se adopta a partir de lo que la investigación avala mediante evidencias empíricas sólidas, intentando superar una intervención basada en supuestos por una práctica avalada por hechos objetivables (Martínez Sánchez, 1995).

Todo ello en un contexto interdisciplinar, al tener que trabajar con distintos profesionales de lo social. Esto pone de relieve que toda intervención socioeducativa debe estar sometida a la lógica de la indagación permanente (poner en cuestión), y en concreto, el educador social debe introducir en su buen hacer profesional tres aspectos: ver, juzgar y actuar a partir del método científico. Esto significa que a través de la investigación debe ser capaz de:

a) Asumir como propio, desde la óptica científica, el ejercicio de profundizar en el conocimiento de los fenómenos y contextos socioeducativos y formativos.

b) Comprender, valorar y juzgar los fenómenos y hechos sociales para optimizar sus proyectos y actuaciones bajo criterios de oportunidad, idoneidad, eficacia, calidad, globalidad y flexibilidad. 
c) Avanzar en su desarrollo profesional como elemento irrenunciable para la mejora de su práctica.

Lo anterior implica desterrar determinadas concepciones academicistas sobre la investigación en Educación Social, alejadas de la realidad y que no aportan un saber hacer aplicado al contexto de intervención socioeducativa. Para estos fines, la investigación socioeducativa es considerada como un medio al servicio de la transformación de los fenómenos, hechos y situaciones educativas, con la intención de ajustar su intervención a las demandas y necesidades reales de los sujetos objeto de intervención y de mejorar la práctica como profesional comprometido con una sociedad democrática, bajo referentes éticos (Fullana, Pallisera, Tesouro y Castro, 2009). Por lo que los métodos de investigación educativa constituyen una subdisciplina científica relacionada con el desarrollo de metodologías de análisis de la realidad social, mostrando las técnicas y los procedimientos adecuados a los objetivos perseguidos en cualquier investigación educativa, de manera que se pueda obtener una descripción, comprensión y explicación científica del objeto/sujeto de estudio con aras a una intervención socioeducativa de calidad.

\section{Contexto de la eXPeriencia docente: DesCripCión de la PLANifiCaCión DIDÁCTICA DE LA ASIGNATURA}

De acuerdo con lo establecido en el marco normativo vigente, y siguiendo las directrices del Libro Blanco, la asignatura de MIE constituye una subdisciplina científica relacionada con el desarrollo de metodologías de análisis de la realidad social, mostrando las técnicas y los procedimientos adecuados a los objetivos perseguidos en cualquier investigación socioeducativa, de manera que se pueda obtener una descripción, comprensión y/o explicación científica del objeto/sujeto de estudio con aras a una intervención socioeducativa de calidad. Así mismo, la investigación y la intervención socio-educativa no se pueden separar ya que la investigación forma parte del proceso de elaboración y acción de la intervención. A continuación presentamos en qué parte de la elaboración de un proyecto de intervención socioeducativa encontramos la investigación, que generará, por tanto, un proyecto de investigación y evaluación y cuyo aprendizaje se desarrolla en la asignatura de MIE. La asignatura se imparte en primer curso de la Titulación de Educación Social, en la Facultad de Ciencias de la Educación y Humanidades del campus de Cuenca (UCLM). Esta asignatura de carácter básica tiene una carga de 160 horas de formación, el equivalente a 6 créditos ECTS. Con esta materia se aporta los aspectos epistemológicos (conceptos y enfoques teóricos) y metodológicos (procedimientos y técnicas) propios del método científico, orientados a la capacitación profesional del educador social, gracias al abordaje de una serie de competencias técnico-profesionales y criterios metodológicos planteados en base a parámetros de calidad y de posicionamiento crítico. Estas pretensiones didácticas y de aprendizaje mantienen una coherencia interna con el plan de estudios que nos hemos dado para el desarrollo profesional del educador social según lo establecido en el programa verifica del grado en Educación Social de la UCLM (Morales y SánchezSantamaría, 2012). 
MIE se integra dentro de la materia de "Métodos de investigación e intervención educativa" que, a su vez, configura el módulo Il titulado, "Bases metodológicas, diseño, programación y evaluación de programas y proyectos socioeducativos" (UCLM, 2008, pp. 98-99). Este módulo aborda de forma conjunta el conocimiento de las bases didácticas y de las metodologías educativas necesarias para el diseño, el desarroIlo y la evaluación de la acción educativa. Esto exige prestar atención al papel que la asignatura juega en el desarrollo formativo del perfil profesional del educador social, buscando en todo momento las conexiones oportunas para garantizar la coherencia y la no duplicidad del contenido bajo una lógica tendente a la coordinación.

El contenido de la asignatura hace especial hincapié en tres aspectos:

- Epistemología: ¿Cómo se elabora el conocimiento científico y bajo qué paradigmas en Educación Social?

- Metodología: ¿Cómo se diseñan y validan distintos instrumentos de investigación en Educación Social, vinculados al diagnóstico y evaluación? ¿Cómo se analizan los datos en Educación Social?

- Difusión y visibilidad: ¿Cómo se comunican los resultados de investigación en Educación Social?

Para dar contenido a estas intenciones educativas, el aprendizaje autónomo y en grupo de los estudiantes y la supervisión continua de ese aprendizaje por parte del profesor tutor, se convierten en pilares fundamentales del proceso de enseñanza y aprendizaje en la asignatura de métodos de investigación en Educación Social (tabla 3).

Tabla 3. Estrategias didácticas para el desarrollo del proceso de aprendizaje

\section{Estrategia didáctica}

\begin{tabular}{ll}
\hline & Realización de prácticas en la parte presencial, relaciona- \\
Prácticas en las sesiones & $\begin{array}{l}\text { do con aspectos básicos de la asignatura, generalmente las } \\
\text { prácticas exigen un tiempo anterior a la sesión de trabajo } \\
\text { presenciales }\end{array}$ \\
& autónomo para poder completarlas con éxito
\end{tabular}

Seminarios

Proyecto grupal con trabajo de campo

Foro de investigación

Examen de conocimientos
Se realiza un seminario de introducción al programa de tratamiento de datos SPSS. El seminario se realiza en grupos reducidos

Se trata de un proyecto de aprendizaje que se realiza de forma grupal. Implica realizar tutorías previas, de seguimiento y final con el profesor

Consiste en la presentación oral del grupo de la síntesis de su proyecto de investigación

Prueba para evaluar las competencias relacionadas con la comprensión, análisis y aplicación de la información teórica que se ofrece al alumnado de cada tema 
Al mismo tiempo el trabajo en equipo, es un elemento clave del proceso de la asignatura, donde los estudiantes trabajaran en grupo de cuatro persona, (Castejón, Capllonch, González y López Pastor, 2009). Las condiciones estructurales, para trabajar de manera cooperativa se orienta (Rue, 2010) principalmente al tipo de actividad que hemos marcado, en nuestro caso el trabajo de investigación, donde el tiempo dedicado a la actividad, todo el cuatrimestre, las instrucciones básicas por parte del profesor acercar del producto a realizar y la evaluación, donde los criterios de calidad que orientarán el informe de evaluación (tabla 4).

Tabla 4. Criterios de calidad del informe de investigación

\section{Dimensiones de evaluación Criterios de evaluación}

I. Diseño: formato y componentes
1. Inclusión de todos los apartados del informe

2. Inclusión de reseñas bibliográficas en el texto

3. Presentación escrita del informe de investigación
II. Contenido: calidad de la información
4. Originalidad e importancia del tema

5. Planteamiento adecuado del problema

6. Relevancia del marco teórico

7. Pertinencia de los objetivos e hipótesis

8. Precisión de las variables

9. Selección adecuada de los participantes

10. Elección de instrumentos en función de los objetivos e hipótesis

11. Construcción precisa de los instrumentos

12. Descripción detallada del procedimiento

13. Pertinencia de los análisis

14. Claridad y coherencia expositiva

15. Amplitud y relevancia de la bibliografía

La conexión entre calificación y evaluación tiene lugar a través de un proceso integrado en el desarrollo de la asignatura, con su aplicación se pretende hacer un proceso realmente formativo y continuo, que forma parte del proceso de aprendizaje. Se asume una evaluación criterial, en relación a los desarrollos alcanzados por los alumnos, en vez de una evaluación normativa basada en lo transmitido. Para ello se han aplicado tres tipos de instrumentos de evaluación sobre el desarrollo competencial del alumnado, tal y como se recoge en la siguiente tabla (5). 
Tabla 5. Denominación, caracterización y momentos de aplicación de la evaluación en función del módulo de contenidos

\begin{tabular}{|c|c|c|c|}
\hline Módulo de contenido & $\begin{array}{c}\text { Denominación } \\
\text { (\% nota) }\end{array}$ & Caracterización & Momentos \\
\hline $\begin{array}{l}\text { Módulo I. } \\
\text { fundamentos básicos } \\
\text { de la investigación en } \\
\text { educación social }\end{array}$ & $\begin{array}{l}\text { Examen } \\
\text { contenidos } \\
(30,0) \%\end{array}$ & $\begin{array}{l}\text { - } \text { Dominio de la materia } \\
\text { - Precisión en las respuestas } \\
\text { - } \text { Claridad expositiva } \\
\text { - Estructuración y relación } \\
\text { de ideas }\end{array}$ & Final \\
\hline \multirow[t]{2}{*}{$\begin{array}{l}\text { Módulo II. } \\
\text { Proceso general de } \\
\text { investigación en } \\
\text { educación social }\end{array}$} & $\begin{array}{l}\text { Proyecto de } \\
\text { Aprendizaje } \\
\text { Basado en } \\
\text { Proyectos } \\
(60,0 \%)\end{array}$ & $\begin{array}{l}\text { - Tutoría sobre el trabajo: } \\
\text { Desde el inicio. } \\
\text { - Evaluación de las sesio- } \\
\text { nes del seguimiento del } \\
\text { grupo a partir de la planti- } \\
\text { Ila de observación } \\
\text { - Informe del proyecto de } \\
\text { investigación por parte de } \\
\text { los alumnos. } \\
\text { - Informe Grupal del traba- } \\
\text { jo de investigación } \\
\text { - Informe Individual de la } \\
\text { asignatura } \\
\text { - Informe del profesor }\end{array}$ & $\begin{array}{l}\text { Inicial, } \\
\text { procesual y } \\
\text { final }\end{array}$ \\
\hline & $\begin{array}{l}\text { Jornadas pre- } \\
\text { profesionales } \\
\text { sobre } \\
\text { Investigación } \\
\text { Socioeducativa } \\
(10,0 \%)\end{array}$ & $\begin{array}{l}\text { - Síntesis del trabajo de in- } \\
\text { vestigación por parte del } \\
\text { profesor } \\
\text { - Informe del profesor so- } \\
\text { bre la exposición oral }\end{array}$ & $\begin{array}{l}\text { Procesual y } \\
\text { final }\end{array}$ \\
\hline
\end{tabular}

A partir de esta cuestión el grupo debe decidir de manera consensuada, qué caso de los 20 propuestos va a investigar. A continuación se muestran algunos ejemplos de casos propuestos a los alumnos (tabla 6).

Tabla 6. Ejemplos de un par de casos propuestos y trabajados por los alumnos

\section{CASO 10. DIAGNÓSTICO DE NECESIDADES DE FAMILIAS INMIGRANTES EN SITUA-} CIÓN DE RIESGO.

COLECTIVO: familias en situación de riesgo.

ÁMBITO: cooperación al desarrollo.

PRESENTACIÓN DEL CASO: habéis creado una asociación de cooperación al desarrollo que os permita intervenir con familias inmigrantes en Cuenca. Para ello, necesitáis saber qué necesidades presenta este colectivo para así desarrollar programas creíbles y reales.

FINALIDAD DE LA INVESTIGACIÓN: desarrollar un diagnóstico de necesidades sobre las familias inmigrantes en situación de riesgo. 


\section{CASO 16. DIAGNÓSTICO DE POSIBILIDADES, INTERESES, CONOCIMIENTOS Y CAPA-} CIDADES PARA LA PROMOCIÓN DEL VOLUNTARIADO DE PERSONAS MAYORES.

COLECTIVO: personas mayores.

ÁMBITO: promoción de voluntariado.

PRESENTACIÓN DEL CASO: la Universidad de Castilla-La Mancha quiere establecer un programa de actividades de voluntariado para los alumnos de la Universidad de Mayores Saramago. Para ello necesita conocer cuáles son las posibilidades, intereses, conocimientos y capacidades de estos alumnos para realizar voluntariado con distintos colectivos y ámbitos de trabajo.

FINALIDAD: realizar un diagnóstico sobre las posibilidades, intereses, conocimientos y capacidades del voluntariado de los alumnos de la Universidad de Mayores.

A continuación se muestra un ejemplo de la organización de Moodle, en concreto del módulo II que por motivos de espacio no es posible mostrar toda la plataforma. No obstante, valga apuntar que, según el manual elaborado ad hoc por el profesor y titulado: "familiarizarse con la plataforma Moodle para MIE, 20011/12" y entregado a los alumnos al inicio del curso, la plataforma se ha organizado en siete bloques: inicial (información relevante sobre la asignatura) e intermedios (2 bloques referidos a los 2 módulos de la asignatura con 1 y 6 temas respectivamente).

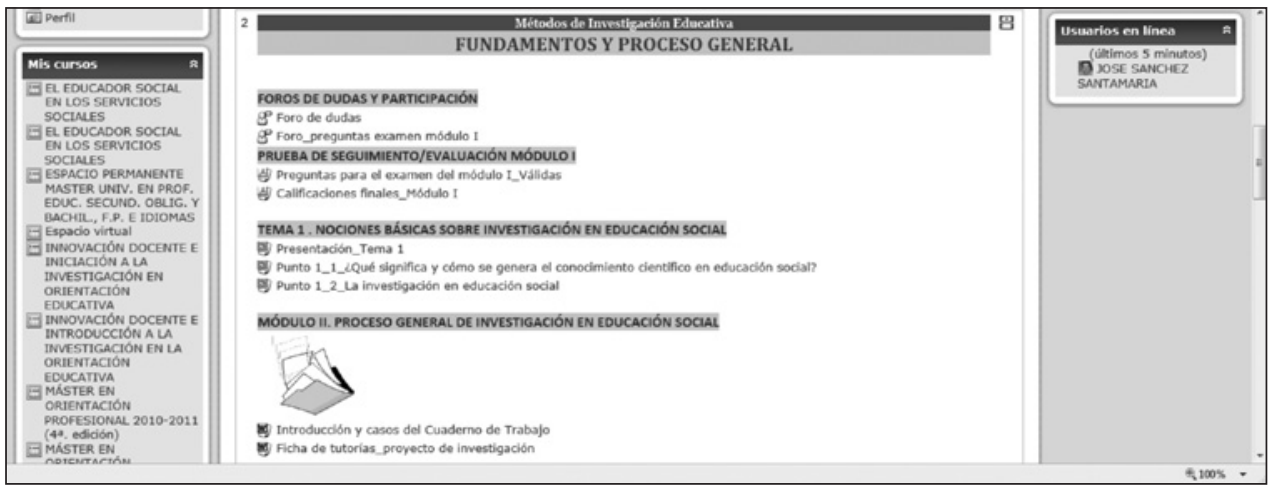

Fuente: https://campusvirtual.uclm.es/course/view.php?id=8217.

Imagen 1. Bloque intermedio de la plataforma Moodle para la asignatura de MIE

\section{OBjetivos, PARTICIPANTES y MÉTOdo}

\subsection{Objetivos}

El objetivo del artículo es conocer y analizar el desarrollo de competencias de investigación educativa mediante el uso de metodologías activas en un entorno B-Learning. Este objetivo general se desarrolla en tres objetivos de trabajo, a saber: a) Describir el proceso didáctico diseñado; $y, b$ ) analizar las percepciones de los estudiantes respecto a al uso de metodologías activas en B-Learning, así como su valoración y su nivel de 
satisfacción sobre las competencias específicas desarrolladas. De este modo, el estudio se centra en recoger evidencias basadas en la investigación que, nos permitan conocer el potencial de las metodologías activas en entornos de B-Learning en la docencia universitaria; $y$, en concreto, en el desarrollo de competencias profesionales vinculadas a la investigación educativa en Educación Social. Además, de abrir nuevos procesos de innovación docente orientados a la promoción del aprendizaje situado del estudiante universitario, de modo que estemos en disposición de aprovechar las posibilidades que puede representar este tipo de combinaciones para la mejora de la calidad.

\subsection{Participantes}

La muestra del estudio respondió a un muestreo no probabilístico de tipo incidental por accesibilidad (Salkind, 1999). La población estaba formada por $\mathrm{N}=79$ estudiantes matriculados en la asignatura en el curso académico 2011/12. Así, de acuerdo a los objetivos de la investigación, la muestra teórica e invitada se limitó a 65 estudiantes (82,3\% del total) de acuerdo a dos criterios: estudiantes acogidos a evaluación continuo-formativa y de matrícula completa. La muestra final fue de $\mathrm{n}=59$ estudiantes, es decir, el 90,7\% del total de la población. De los cuales, el 76,3\% eran mujeres y el 23,7\% hombres, con una edad media de 19,6 años $(S x=2,457)$, ya que el rango de edad era de 18 años a 35 años. Esta selección muestral contemplaba un nivel de confianza del $90,0 \%$ (1,65 sigmas) y con un error muestral \pm 5 en el supuesto de un muestreo aleatorio simple. No obstante, se puede afirmar que la muestra cumple con los criterios de calidad suficientes, válidos y representativos para poder obtener evidencias relevantes.

\subsection{Método del estudio}

El método utilizado es el de encuesta con vocación descriptivo-exploratoria. Para la recogida de información se ha utilizado un cuestionario ad hoc titulado "Cuestionario de Percepciones del Alumnado Universitario sobre el Desarrollo de Competencias de Investigación Educativa" (CUPAUDECIE_v01). Es un cuestionario validado, mediante 10 jueces y una experiencia piloto. Siguiendo una escala tipo Likert de $1 \mathrm{a}$ 5 (de $1=$ en total desacuerdo hasta $5=$ totalmente de acuerdo) (Morales, Urosa y Blanco, 2003), el cuestionario se compone de 4 dimensiones de análisis con 33 variables, con un Alpha de Cronbach de .811, superior al valor mínimo de 0,70 que se utiliza como referente para determinar la consistencia interna.

Tabla 7. Cuestionario de Percepciones del Alumnado Universitario sobre el DesarroIlo de Competencias en Investigación Educativa: Dimensiones, variables y fiabilidad

\begin{tabular}{lcc}
\hline Dimensión & Variables & Fiabilidad $(\alpha)$ \\
\hline I. Datos de identificación & 3 & No procede \\
\hline II. Combinación didáctica: ABP y B-Learning & 13 & .835 \\
\hline III. Competencias específicas & 11 & .812 \\
\hline IV. Estrategias didácticas & 6 & .730 \\
\hline
\end{tabular}

Fuente: Elaboración propia, a partir del Cuestionario de Percepciones del Alumnado Universitario sobre el Desarrollo de Competencias en Investigación Educativa, 2012. 
Para la validación del instrumento, se consulto a 10 expertos en docencia universitaria y desarrollo de competencias vinculadas con los métodos de investigación educativa de diversas universidades españolas: Castilla-La Mancha (2), Valencia (3), Madrid (3) y Barcelona (2). Se siguió la recomendación de Lawshe (1975), cuando establece una razón de validez mínima de 0,62 con 10 expertos para reducir los efectos del azar. El proceso seguido fue el habitual: en primer lugar, se identifico a los expertos, estableciendo contacto con ellos mediante el envío de un correo electrónico con la documentación necesaria para hacer la validación. A esta fase, fueron invitadas a partir un total de 18 expertos, de los respondieron afirmativamente 10 de ellos; en segundo lugar, elaboración de los expertos de la plantilla de respuesta (tabla 8), mediante una escala tipo Likert de 1 a 4 (donde 1 =en total desacuerdo, 2=desacuerdo, $3=$ de acuerdo; 4= totalmente de acuerdo). Además de ello, los expertos tenían la posibilidad de aportar cuantos comentarios y/o observaciones estimaran oportunas; $y$, en tercer lugar, se recibieron los documentos con las valoraciones de los jueves. Se tomaron en consideración para su mantenimiento en el instrumento los ítems con un grado de concordancia superior al $80,0 \%$.

Tabla 8. Descriptivos: Validación expertos

\begin{tabular}{lcc}
\hline \multicolumn{1}{c}{ Indicador de análisis } & M & sx \\
\hline I. Relación: fundamentación teórica y contenido del instrumento & 3,70 &, 823 \\
\hline II. Correspondencia: dimensiones e ítems del instrumento & 3,60 &, 843 \\
\hline III. Claridad y accesibilidad: lenguaje & 4,00 &, 816 \\
\hline IV. Adecuación: número total de ítems & 3,50 &, 512 \\
\hline V. Recomendación: quitar y/o ajustar dimensiones y/o ítems & 1,50 &, 707 \\
\hline
\end{tabular}

Fuente: Datos extraídos de las guías de valoración del procedimiento de juicio de expertos, 2012.

El procedimiento general de recogida de la información se desarrolló desde el inicio de las sesiones el (1 de mayo) hasta unos días antes al cierre de las actas (12 de junio). Para el análisis de los datos se ha utilizado el programa informático SPSS versión 19, bajo licencia de la Universidad de Castilla-La Mancha (2012). Por último, y desde el punto de vista del metodológico, este estudio presenta dos limitaciones que deben ser consideradas para una adecuada interpretación de los datos presentados, a saber: a) la propia naturaleza del trabajo como estudio de caso, hace que se tenga que comprender más en términos de buena práctica docente que de una investigación propiamente dicha. Este aspecto limita la validez ecológica; b) al tratarse de un estudio descriptivo-exploratorio, no se aportan evidencias que permita explicar y/o comprender el fenómeno de estudio. Por ello, en próximos estudios retomaremos estas cuestiones, ampliando la muestra y teniendo en cuenta los criterios necesarios para hacer inferencias estadísticas. Del mismo modo, se apostará por un diseño mixto, con la doble orientación cuantitativa y cualitativa, haciendo uso de la triangulación de fuentes, técnicas y actores. 


\section{Resultados sobre el uso de metodologías activas en un CONTEXto B-LEARNING}

\subsection{Utilidad percibida}

En términos generales, los alumnos valoran de forma destacable la utilidad del uso de metodologías activas en un contexto B-learning. Así pues, hay tres aspectos que sobresalen sobre el resto: a) que permite un seguimiento más completo de la asignatura $(M=4,19 ; S x=, 779)$; b) que promueve el aprendizaje cooperativo $(M=4,08$; $S x=, 836) ; y, c)$ que facilita el trabajo en equipo $(M=4,02 ; S x=, 841)$. La única cuestión que presenta un valor a estudiar es el que se refiere a si se facilita la planificación del estudiante $(M=2,66, S x: 1,060)$. Aunque existe una diversidad mayor de opiniones, en relación al resto de indicadores sobre la utilidad percibida, se puede afirmar que este valor se debe al peso real que todavía tiene, en una Universidad presencial, la planificación de lo que ocurre dentro del aula.

Tabla 9. Descriptivos: Utilidad percibida sobre el uso de metodologías activas en un contexto B-Learning

\begin{tabular}{|c|c|c|c|c|c|c|c|}
\hline \multirow{2}{*}{ Dimensión I: variables } & \multirow{2}{*}{$M_{0}$} & \multirow{2}{*}{$\mathbf{M}$} & \multirow{2}{*}{ Sx } & \multicolumn{2}{|c|}{ Rango } & \multicolumn{2}{|c|}{ Género } \\
\hline & & & & Mín. & Máx. & $\mathbf{F}$ & Sig. \\
\hline $\begin{array}{l}\text { Facilita mi proceso de } \\
\text { aprendizaje }\end{array}$ & 4 & 3,86 & ,730 & 3 & 5 & ,675 & ,415 \\
\hline $\begin{array}{l}\text { Aumenta la responsabilidad en } \\
\text { el aprendizaje }\end{array}$ & 4 & 3,63 & ,849 & 1 & 5 & ,805 &, 373 \\
\hline $\begin{array}{l}\text { Promueve el aprendizaje } \\
\text { colaborativo }\end{array}$ & 4 & 4,08 & ,836 & 1 & 5 &, 064 & 801 \\
\hline $\begin{array}{l}\text { Permite alcanzar mejor los } \\
\text { objetivos de aprendizaje }\end{array}$ & 4 & 3,39 & ,929 & 1 & 5 & 1,596 &, 212 \\
\hline $\begin{array}{l}\text { Facilita la comunicación con } \\
\text { el profesor }\end{array}$ & 4 & 3,46 & ,934 & 1 & 5 & 4,890 & ,031 \\
\hline $\begin{array}{l}\text { Permite trabajas competencias } \\
\text { emocionales }\end{array}$ & 4 & 3,64 & ,886 & 2 & 5 & 2,791 &, 100 \\
\hline Estimula el pensamiento crítico & 4 & 3,83 & ,950 & 1 & 5 & 025 & 874 \\
\hline $\begin{array}{l}\text { Potencia la iniciativa y espíritu } \\
\text { emprendedor }\end{array}$ & 3 & 3,39 & 1,017 & 1 & 5 & ,463 & ,499 \\
\hline $\begin{array}{l}\text { Permite un seguimiento más } \\
\text { completo de la asignatura }\end{array}$ & 4 & 4,19 &, 776 & 2 & 5 & ,301 &, 585 \\
\hline $\begin{array}{l}\text { Aumenta la participación del } \\
\text { estudiante }\end{array}$ & 4 & 3,46 & ,934 & 1 & 5 &, 657 &, 421 \\
\hline Facilita el trabajo en equipo & 4 & 4,02 & 841 & 2 & 5 & 1,296 & 260 \\
\hline $\begin{array}{l}\text { Potencia la motivación a la } \\
\text { hora de aprender }\end{array}$ & 4 & 3,73 & ,944 & 1 & 5 & 2,281 &, 136 \\
\hline $\begin{array}{l}\text { Facilita la planificación del } \\
\text { estudiante }\end{array}$ & 2 & 2,66 & 1,060 & 1 & 5 & 2,299 &, 135 \\
\hline
\end{tabular}

Fuente: Elaboración propia, a partir del Cuestionario de Percepciones del Alumnado Universitario sobre el Desarrollo de Competencias en Investigación Educativa, 2012. 
A continuación, se detallan los porcentajes de las respuestas dadas por los alumnos participantes en el estudio (gráfico 1). En lo que respecta a: "potencia la motivación a la hora de aprender" y "facilita la planificación del estudiante" y más del $75,0 \%$ y del $45,0 \%$, respectivamente, entienden que nada o poco. Por su parte, el resto de valores muestran datos más satisfactorios.

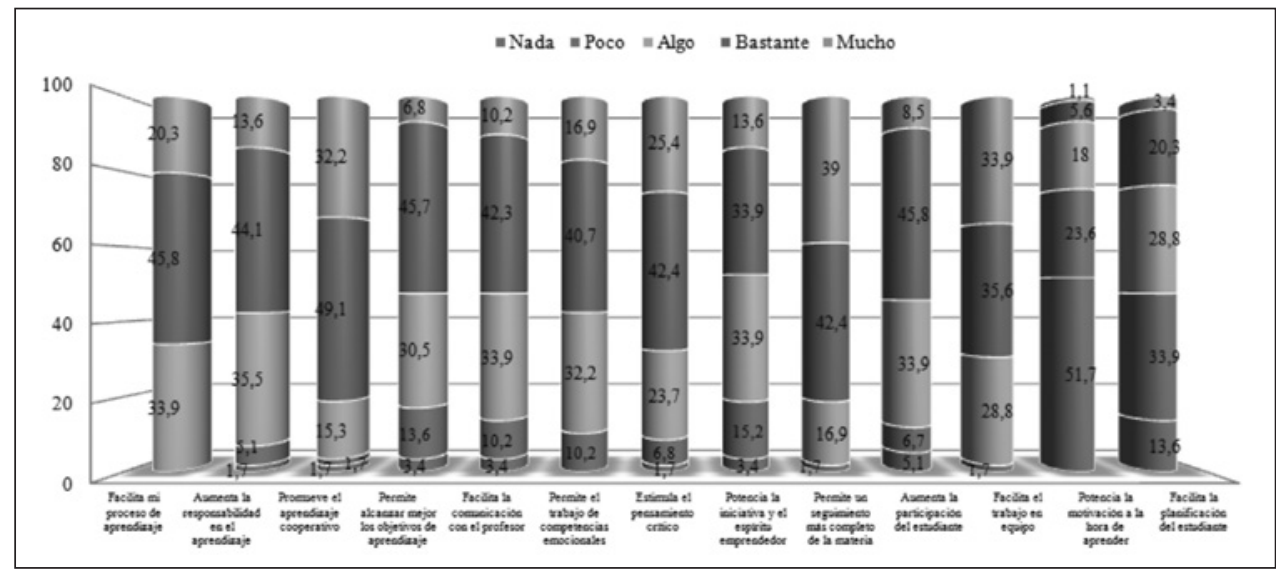

Fuente: Elaboración propia, a partir del Cuestionario de Percepciones del Alumnado Universitario sobre el Desarrollo de Competencias en Investigación Educativa, 2012.

Gráfico 1. Utilidad percibida sobre el uso de metodologías activas en B-Learning (\%)

\subsection{Desarrollo competencial percibido}

Existe un acuerdo generalizado sobre el papel destacado del uso de metodologías activas en B-Learning. Como se aprecia en la tabla 11, los promedios dados por los alumnos son superiores a 3, es decir, de acuerdo, incluso competencias como: "capacidad para utilizar el paradigma de investigación acorde al problema a investigar", "capacidad para elaborar e interpretar documentos e informes de investigación" o "capacidad de utilizar distintos recursos de búsqueda de datos" son muy valorados. En cierta manera, estos datos son bastante coherentes con el trabajo realizado en el aula, ya que el uso de tareas auténticas sobre las cuales se van trabajando los contenidos de los temas, unido a la necesidad de incorporar recursos tecnológicos potenciados por el B-learning, son responsables de estas valoraciones.

Tabla 10. Descriptivos: Desarrollo competencial percibido a partir de metodologías activas en B-Learning

\begin{tabular}{|c|c|c|c|c|c|}
\hline \multirow{2}{*}{ Dimensión II: variables } & \multirow{2}{*}{$M_{0}$} & \multirow{2}{*}{$\mathbf{M}$} & \multirow{2}{*}{ Sx } & \multicolumn{2}{|c|}{ Rango } \\
\hline & & & & Mín. & Máx. \\
\hline $\begin{array}{l}\text { Ser capaz de detectar y valorar de } \\
\text { problemas de investigación }\end{array}$ & 4 & 3,88 & ,745 & 2 & 5 \\
\hline
\end{tabular}


Tabla 10. Descriptivos: Desarrollo competencial percibido a partir de metodologías activas en B-Learning (continuación)

\begin{tabular}{|c|c|c|c|c|c|}
\hline \multirow{2}{*}{ Dimensión II: variables } & \multirow{2}{*}{$M_{0}$} & \multirow{2}{*}{$\mathbf{M}$} & \multirow{2}{*}{ Sx } & \multicolumn{2}{|c|}{ Rango } \\
\hline & & & & Mín. & Máx. \\
\hline $\begin{array}{l}\text { Ser capaz de utilizar un paradigma de } \\
\text { investigación acorde al problema a } \\
\text { investigar }\end{array}$ & 4 & 4,39 &, 558 & 3 & 5 \\
\hline $\begin{array}{l}\text { Ser capaz de utilizar distintos recursos de } \\
\text { búsqueda de datos }\end{array}$ & 4 & 4,41 &, 591 & 3 & 5 \\
\hline $\begin{array}{l}\text { Ser capaz de valorar la coherencia y la } \\
\text { calidad de informes, publicaciones de } \\
\text { investigación }\end{array}$ & 4 & 4,15 & ,805 & 2 & 5 \\
\hline $\begin{array}{l}\text { Ser capaz de aplicar distintas estrategias } \\
\text { de recogida de información acorde al } \\
\text { problema a investigar }\end{array}$ & 3 & 3,51 & ,858 & 2 & 5 \\
\hline $\begin{array}{l}\text { Ser capaz de aplicar distintas estrategias } \\
\text { de análisis acorde al problema a investigar }\end{array}$ & 3 & 3,59 & 1,069 & 1 & 5 \\
\hline $\begin{array}{l}\text { Ser capaz de elaborar e interpretar de } \\
\text { documentos e informes de investigación }\end{array}$ & 4 & 3,53 & ,817 & 2 & 5 \\
\hline $\begin{array}{l}\text { Ser capaz de utilizar distintos recursos } \\
\text { informáticos para el análisis estadístico }\end{array}$ & 3 & 3,10 & 1,012 & 1 & 5 \\
\hline $\begin{array}{l}\text { Ser capaz de promover procesos de } \\
\text { mediación educativa orientados a } \\
\text { la convivencia y a la resolución de } \\
\text { conflictos. }\end{array}$ & 4 & 4,02 & ,900 & 2 & 5 \\
\hline $\begin{array}{l}\text { Ser capaz de diseñar, implementar y } \\
\text { evaluar estrategias, planes, programas, } \\
\text { proyectos y acciones socioeducativas en } \\
\text { diversos contextos y ámbitos. }\end{array}$ & 4 & 3,85 & ,997 & 1 & 5 \\
\hline $\begin{array}{l}\text { Ser capaz de asesorar y acompañar de } \\
\text { forma educativa a personas y grupos }\end{array}$ & 4 & 3,64 & ,846 & 1 & 5 \\
\hline
\end{tabular}

Fuente: Elaboración propia, a partir del Cuestionario de Percepciones del Alumnado Universitario sobre el Desarrollo de Competencias en Investigación Educativa, 2012.

\subsection{Estrategias didácticas y uso de metodologías activas en un contexto B-learning}

Como se aprecia en la tabla 11, en términos generales, el uso de metodologías activas se relaciona de forma significativa con clases teóricas, trabajo en grupo e individual que con otro tipo de estrategias didácticas utilizadas en MIE. Sobresale, el hecho de que en el caso de las tutorías y seminarios les otorguen una valoración baja, se debe a: en el primer caso, no existen tutorías on-line programas en la guía docente, todas se desarrollan de forma presencial. No identifican la respuesta del profesor a correos de los alumnos como tutorías; y, en el segundo caso, el seminario se ha centrado en actividades que han requerido poca presencia del B-learning. 
Tabla 11. Descriptivos: Importancia de las metodologías activas en un contexto B-Learning

\begin{tabular}{lcccccc}
\hline \multicolumn{1}{c}{ Dimensión I: variables } & & & & \multicolumn{2}{c}{ Rango } \\
\cline { 6 - 7 } & $\mathbf{M}_{0}$ & $\mathbf{M}$ & Sx & & Mín. & Máx. \\
\hline Clases teóricas & 5 & 4,47 &, 598 & 3 & 5 \\
\hline Tutorías & 3 & 3,58 &, 747 & 2 & 5 \\
\hline Clases prácticas & 3 & 2,71 &, 696 & 2 & 4 \\
\hline Trabajo en grupo & 5 & 4,10 &, 865 & 2 & 5 \\
\hline Trabajo individual & 5 & 4,07 & 1,015 & 1 & 5 \\
\hline Seminarios & 3 & 2,83 &, 791 & 2 & 5 \\
\hline
\end{tabular}

Fuente: Elaboración propia, a partir del Cuestionario de Percepciones del Alumnado Universitario sobre el Desarrollo de Competencias en Investigación Educativa, 2012.

A continuación se muestran los porcentajes globales sobre la satisfacción percibida respecto al uso de las distintas metodologías activas en B-learning (gráfico 2).

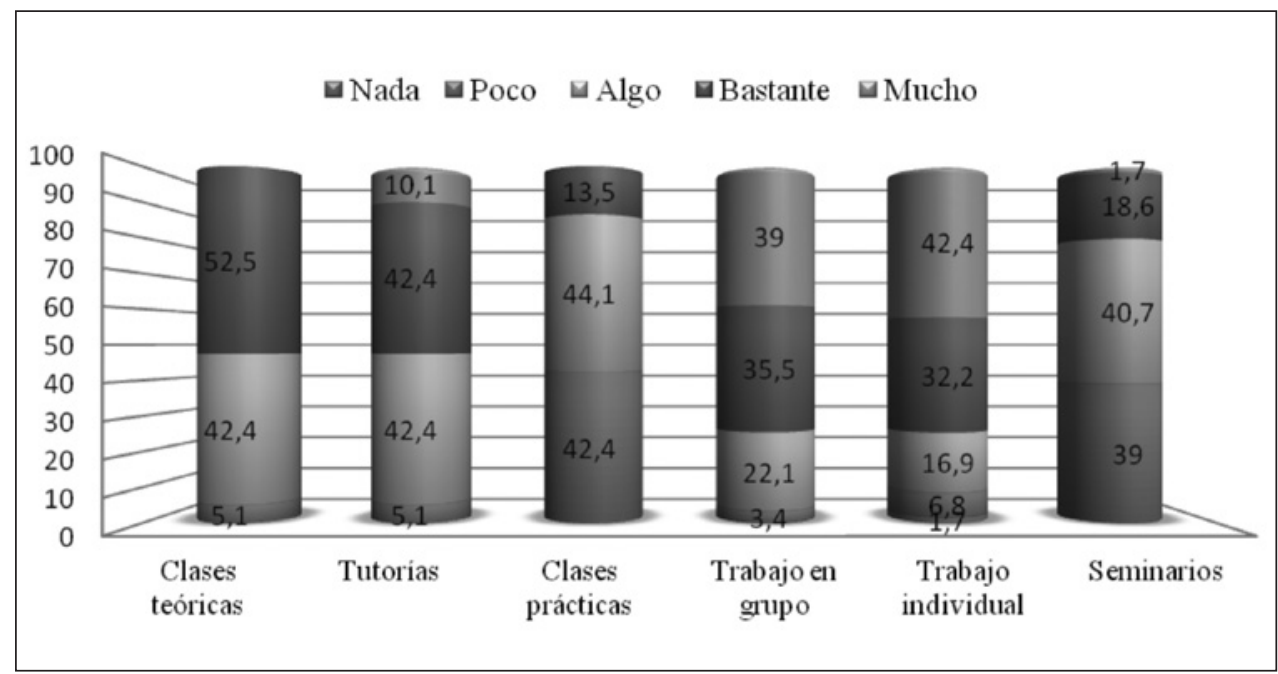

Fuente: Elaboración propia, a partir del Cuestionario de Percepciones del Alumnado Universitario sobre el Desarrollo de Competencias en Investigación Educativa, 2012.

Gráfico 2. Uso de metodologías activas en contexto B-learning (\%)

\subsection{Valoración global sobre el uso de metodologías activas en un contexto B-Learning}

Los alumnos manifiestan un grado satisfacción global medio, con un valor promedio de 3,39. Aunque el apoyo recibido y el desarrollo competencial son muy bien valorados, la carga de trabajo y la dificultad encontrada son percibidos con menor valor. 
Tabla 12. Descriptivos: valoración global

\begin{tabular}{lcccccc}
\hline \multicolumn{1}{c}{ Dimensión I: variables } & & & & \multicolumn{2}{c}{ Rango } \\
\cline { 7 - 8 } & $\mathbf{M}_{\mathbf{0}}$ & $\mathbf{M}$ & $\mathbf{S x}$ & Mín. & Máx. \\
\hline Dificultad encontrada & 4 & 3,39 & 1,145 & 1 & 5 \\
\hline Carga de trabajo & 4 & 3,47 & 1,194 & 1 & 5 \\
\hline Apoyo recibido & 5 & 4,54 &, 816 & 2 & 5 \\
\hline Desarrollo competencial & 5 & 4,34 & 1,044 & 1 & 5 \\
\hline \multicolumn{2}{l}{ Satisfacción global } & & & & & \\
\hline
\end{tabular}

Fuente: Elaboración propia, a partir del Cuestionario de Percepciones del Alumnado Universitario sobre el Desarrollo de Competencias en Investigación Educativa, 2012.

\section{Conclusiones}

Con este artículo se ha perseguido mostrar y analizar las percepciones de los alumnos sobre su aprendizaje competencial mediante el uso de metodologías activas en un contexto B-learning, en la asignatura de MIE, dentro del Plan de estudios del Grado de Educación Social de la UCLM, en el Campus Universitario de Cuenca (España). Así pues, considerando las sugerencias de Zabalza y Zabalza Cerdeiriña (2010) acerca de una enseñanza universitaria de calidad, de la experiencia realizada cabe destacar, al menos, tres aspectos:

a) Respecto a los aprendizajes, podemos destacar una mejora sustancial de los alumnos para seleccionar y gestionar información, así como la optimización de la toma de decisiones, la capacidad reflexiva y crítica, tanto del contenido como del propio aprendizaje y la capacidad de aprender de forma autónoma y significativa.

b) el rol del profesor como guía y del alumnado como protagonista de su aprendizaje; metodológicas, con una organización modular, continua y coordinada que atienda la diversidad de los ritmos de aprendizaje, así como el impulso de métodos activos con el apoyo de recursos didácticos orientados a la tarea; $y$, de evaluación, mediante tareas auténticas y un sistema de evaluación continua y formativa para estimular el aprendizaje autónomo y responsable.

c) El uso de soporte digital ha permitido introducir mejoras en los tiempos y modos de interacción, a la vez que ha facilitado los procesos de evaluación formativa.

Por su parte, también se pueden establecer, al menos, cuatro limitaciones o aspectos susceptibles de mejora, a saber:

a) Se debe concretar y ajustar de forma adecuada la información inicial sobre la realización del proyecto, de modo que se atienda a problemas de seguridad, confianza, etc., que puedan tener los alumnos.

b) La dimensión emocional debe ser estudiada. Emociones negativas como la frustración, el estrés o el cansancio pueden influir de forma importante en la actitud del alumno a la hora de afrontar la tarea. 
c) Respecto a la evaluación es necesario establecer criterios de calidad que permitan extraer evidencias válidas, así como mejorar el modo en cómo se evalúan los criterios competenciales de desempeño, para avanzar en el desarrollo dimensional y criterial de las competencias universitarias, de acuerdo a criterios de calidad, integralidad, confiabilidad y justicia.

De la experiencia llevada a cabo, y en lineamiento con el proceso de investigación-innovación docente del que forma parte este trabajo, se abren diversos interrogantes que requieren seguir profundizando sobre la cuestión aquí tratada, a saber:

a) Profundizar en el estudio de las implicaciones del proyecto de investigación socioeducativa como marco idóneo para el desarrollo competencial del alumnado, de modo que se pueda articular una propuesta global bajo el mismo caso con otras asignaturas; de modo que se pueda trabajar de forma coordinada, a modo de aprendizaje basado en problemas; promoviendo la coordinación docente y el aprendizaje globalizado y situado (Morales y Sánchez-Santamaría, 2011).

b) Fortalecer, todavía más, el trabajo cooperativo en un contexto B-learning, apostando por las tareas auténticas mediante casos que articulen un conjunto de actividades y que impliquen al estudiante de forma colectiva.

c) Delimitación de los niveles e indicadores de desarrollo competencial en términos de progresivo y terminal, de modo que oriente el diseño de e-rúbricas de evaluación aplicables a la evaluación del proyecto de investigación socioeducativa (Sánchez-Santamaría, 2011).

d) Identificación y validación de criterios de evaluación para la selección de evidencias de calidad sobre las implicaciones en el rendimiento académico del alumno, respecto a su desarrollo competencial (Manzanares y Sánchez-Santamaría, 2012).

e) Abrir procesos de reflexión para articular un mayor protagonismo del alumnado, en el sentido de avanzar hacia metodologías participativas (Morales, 2010, 2011) y evaluaciones auténticas (Sánchez-Santamaría, 2011).

En definitiva, esta combinación: metodologías activas + B-Learning, nos exige contemplar el papel que el alumno debe tener en su proceso de construcción del conocimiento, en el sentido de que tenemos que avanzar en superar las limitaciones autoimpuestas como: no es posible trabajar las competencias de trabajo en equipo o cómo vamos a poder comprobar que el alumno es quién hace las tareas, etc., y ser capaces de poder aplicar las potencialidades que esta combinación nos concede, incluso, dando un papel más protagonista al alumno, en por ejemplo, la gestión del entorno virtual de aprendizaje, en la negociación de la guía docente dentro de los límites de nuestro plan de estudios, entre otros.

\section{REFERENCIAS BIBLIOGRÁFICAS}

ALONSO, F.; LÓPEZ, G.; MANRIQUE, D. y VIÑES, J.M. (2005). An instructional model for web-based e-learning education with a blended learning process approach. British Journal of Educational Technology, 36 (2), 217-235. 
ASEDES (2004). Documentos profesionalizadores. Educación Social. Disponible en: http://www.eduso.net/archivo/docdow.php?id=143.

BAUTISTA, G.; BORGES, F. y FORÉS, A. (2006). Didáctica universitaria en entornos virtuales de enseñanza- aprendizaje. Madrid: Narcea.

CASTEJÓN, J.; CAPLLONCH, M.; GONZÁLEZ, N. y LÓPEZ PASTOR, V.M. (2009). Técnicas en instrumentos de evaluación. En V.M. López Pastor (dir.), Evaluación Formativa y Compartida en Educación Superior, propuestas, técnicas, instrumentos y experiencias (pp. 65-90). Madrid: Narcea.

COLE, J.R. y HELEN, C. (2007). Using Moodle: teaching with the popular open source course management system. New York: O'Reilly Media.

FULLANA, E. (1999). Els àmbits de treball de l'educador social. Málaga. Aljibe.

FULLANA, J.; PALLISERA, M.; TESOURO, M. y CASTRO, M. (2009). Professional placement and professional recognition of social education graduates in Spain. A tenyear balance. Social Work Education. The International Journal, 28 (4), 336-350.

GINÉS MORA, J. (2004). La necesidad del cambio educativo para la sociedad del conocimiento. Revista Iberoamericana de Educación, 35, 13-37.

GÓMEZ SERRA, M. (2003). Aproximación conceptual a los sectores y ámbitos de intervención de la Educación Social. Pedagogía Social. Revista Interuniversitaria, 10, segunda época, 233-251.

GONZÁLEZ-VIDEGARAY, M.C. (2007). Evaluación de la reacción de alumnos y docentes en un modelo mixto de aprendizaje para Educación Superior. Revista electrónica de Investigación y Evaluación Educativa (RELIEVE), 13 (1). Disponible en: http://www.uv.es/RELIEVE/v13n1/RELIEVEv13n1_4.htm.

IBM. IBM SPSS Statistics [CD-ROM on-line]. Versión 19. New York: IBM, 2010.

JÄRVELÄ, S. (2006). Personalised Learning? New Insights into Fostering Learning Capacity. En OECD-CERI (eds.), Personalising Education (pp. 31-46). Paris: OECD/ CERI.

LAWSHE, C.H. (1975). A quantitative approach to content validity. Personnel Psychology, 28, 563-575.

LÓPEZ ZAGUIRRE, R. (2002). El Educador Social: Salidas profesionales en la actualidad. Valencia: Escola d'Estiu de l'Educació Social.

LUQUE, P.A. (1998). Ámbitos de acción en educación social y animación sociocultural. En G. Pérez Serrano (coord.), Contexto cultural y socioeducativo de la educación social (pp. 261-291). Sevilla: Universidad de Sevilla.

MAIZTEGUI, C. y SANTIBÁÑEZ, R. (coord.) (2002). El futuro del educador. Perfiles profesionales y adaptación de los pedagogos y educadores sociales a una sociedad en cambio. Bilbao: Universidad de Deusto, 2002.

MANZANARES, A. y SÁNCHEZ SANTAMARÍA, J. (2012). La dimensión pedagógica de la evaluación por competencias y la promoción del desarrollo profesional en el estudiante universitario. Revista Iberoamericana de Evaluación Educativa, 5 (1), 187 202. Disponible en: http://www.rinace.net/riee/numeros/vol5-num1_e/art13.pdf.

MARTÍNEZ SÁNCHEZ, A. (1995). La investigación como fundamento de la intervención social. En P. González, Y. Medina y S. de la Torre (coords.), Didáctica general: modelos y estrategias para la intervención social (pp. 37-82). Madrid: Universitas. 
MATA SALVADOR, F. (1998). Intervención educativa en situaciones de inadaptación social. Granada: IC.

MEC (2006). Propuestas para la renovación de las metodologías educativas en la Universidad. Madrid. Disponible en: http://sestud.uv.es/varios/ope/PROPUESTA_ RENOVACION.pdf.

MORALES, P.; UROSA, B. y BLANCO, A. (2003). Construcción de escalas de actitudes tipo Likert. Madrid: La Muralla.

MORALES, S. (2010). El Practicum en Educación Social: El portafolio como instrumento de seguimiento y evaluación del aprendizaje de los alumnos. Revista Docencia e Investigación, 20, 33-58.

MORALES, S. (dir.) (2011). Nuevos contextos de enseñanza y aprendizaje en el Espacio Europeo de Enseñanza Superior. Buenos Aires: Miño y Dávila.

MORALES, S. y SÁNCHEZ-SANTAMARÍA, J. (2011). La pregunta de investigación como estímulo del proceso de enseñanza y aprendizaje en la asignatura de Métodos de Investigación en Educación Social. En J. Lirio y E. Portal (dirs.), Educación Social. Materiales y estrategias didácticas de apoyo para el título de grado (pp. 149-163). Talavera de la Reina: Universidad de Castilla-La Mancha.

MORALES, S. y SÁNCHEZ-SANTAMARÍA, J. (2012). Una experiencia docente sobre aprender a investigar en educación social: diseño y desarrollo de proyectos de investigación socioeducativa. Enseñanza \& Teaching: Revista Interuniversitaria de Didáctica, 30 (1), 181-204.

PETRUS, A. (1994). Los estudios universitarios de educación social. Estrategias de actuación. En A. Muñoz. (ed.), El educador social: profesión y formación universitaria (pp. 45-60). Madrid: Popular.

PETRUS, A. (2000). Nuevos ámbitos en educación social. En M. Romans, A. Petrus y J. Trilla (coords.), De profesión educador/a social (pp. 61-82). Barcelona: Paidos.

PETRUS, A. (coord.) (1997). Pedagogía Social. Barcelona: Ariel.

RIERA ROMANII, J. (1996). Concepte, formació i professionalització de l'educador social, el treballador social i el pedagog social. Bellaterra: Universitat Autònoma de Barcelona. Tesis doctoral inédita.

RODRÍGUEZ DAMIÁN, A. et al. (2009). Las TIC en la Educación Superior: estudio de los factores intervinientes en la adopción de un LMS por profesores innovadores. Revista Latinoamericana de Tecnología Educativa, RELATEC, 8 (1), 35-51. Disponibel en: http://www.campusvirtual.unex.es/cala/editio.

RUÉ, J. (2010). El aprendizaje Autónomo en Educación Superior. Madrid: Narcea.

RUIZ, A. (coord.) (2003). Educación social. Viejos usos y nuevos retos. Valencia: Universitat de València.

SÁEZ, J. (coord.) (1994). El educador social. Murcia: Universidad de Murcia.

SÁEZ, J. (1997). La construcción de la pedagogía social: algunas vías de aproximación. En A. Petrus (coord.), Pedagogía social (pp. 40-67). Barcelona: Ariel.

SALKIND, N.J. (1999). Métodos de investigación. México D.F.: Pearson Educación.

SALMERÓN, H.; RODRÍGUEZ, S. y GUTIÉRREZ, C. (2010). Metodologías que optimizan la comunicación en entornos de aprendizaje virtual. Comunicar XVII, 34, 163-171. 
SÁNCHEZ-SANTAMARÍA, J. (2011). Evaluación de los aprendizajes universitarios: una comparación sobre sus posibilidades y limitaciones en el Espacio Europeo de Educación Superior. Revista de Formación e Innovación Educativa Universitaria (REFIEDU), 4 (1), 40-54. Disponible en: http://webs.uvigo.es/refiedu/Refiedu/ Vol4_1/Refiedu\%204.1.4.pdf.

SILVA, G.J. y RAMOS, W. (2011). O Ambiente Virtual de Aprendizagem (AVA) como Potencializador da Autonomia do Estudante: Estudo de Caso na UAB-UnB. Revista Iberoamericana de Evaluación Educativa, 4 (2), 92-106. Disponible en: http:// www.rinace.net/riee/numeros/vol4-num2/art5.pdf.

UCEDA ANTOLÍN, J. y BARRO, S. (2009). Las TIC en el sistema universitario español. Madrid: CRUE.

VILLA, A. (coord.) (2004). Libro Blanco del título de grado en pedagogía y educación social. Madrid: ANECA. Disponible en: http://www.aneca.es/var/media/150392/ libroblanco_pedagogia1_0305.pdf.

VILLA, A. y POBLETE, M. (2007). Aprendizaje basado en competencias. Bilbao: Mensajero.

ZABALZA, M. y ZABALZA CERDEIRIÑA, M.A. (2010). Planificación de la docencia en la universidad. Elaboración de las Guías Docentes de las Materias. Madrid: Narcea. 